\title{
Kinks Free Charged Particles from Flux Ropes
}

\author{
Charged particles accelerated by magnetic reconnection in astrophysical \\ plasmas should get stuck in flux ropes. Simulations show that kink \\ instabilities set them free.
}

By Marric Stephens

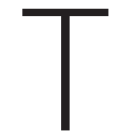
he energies of electrons and protons detected in solar flares and in Earth's magnetotail indicate that the particles are accelerated into nonthermal energy distributions. The acceleration mechanism is widely thought to involve magnetic reconnection-the abrupt reconfiguration of magnetic-field topology-but previous simulations of the phenomenon had failed to reproduce observations. Now, Qile Zhang at Los Alamos National Laboratory, New Mexico, and colleagues have rectified that problem by incorporating into their simulations an effect called the flux-rope kink instability [1]. Zhang says that this instability might also play a role in particle emission from accretion disks and active galactic nuclei.

Previous models linked the particle-energy distribution with magnetic reconnection via the so-called Fermi-acceleration mechanism, where electrons and protons are accelerated by field-line contraction. But these models also predict that
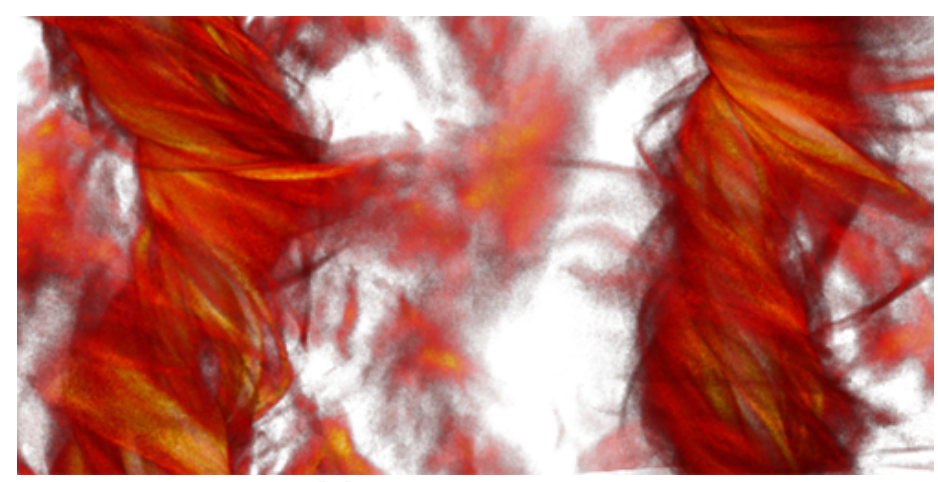

Credit: Q. Zhang et al. [1] magnetic reconnection generates twisted bundles of field lines called flux ropes, which should trap the particles away from Fermi-acceleration regions. Under certain conditions, flux ropes can overlap and disrupt each other, creating field-line chaos that allows the particles to escape. The problem is that the conditions that aid this escape and the conditions under which the Fermi mechanism is most efficient seem to be mutually exclusive: Flux-rope disruption requires large magnetic fields perpendicular to the reconnection plane, while an efficient Fermi mechanism requires weak ones.

In their new simulations, Zhang and colleagues find that field-line chaos can also develop in the weak-field regime if the flux ropes reach a critical length. Then, the flux ropes develop kink instabilities, which deform the flux surfaces and then tear them up. Once freed from the flux ropes, the particles are accelerated by the Fermi mechanism, gaining an energy distribution that closely matches observations.

Marric Stephens is a Corresponding Editor for Physics based in Bristol, UK.

\section{REFERENCES}

1. Q. Zhang et al., "Efficient nonthermal ion and electron acceleration enabled by the flux-rope kink instability in 3D nonrelativistic magnetic reconnection," Phys. Rev. Lett. 127, 185101 (2021). 